\title{
Ritmos em Performance: Análise métrica do Párodo de As Suplicantes de Ésquilo
}

Rhythms in Performance: Metrical analysis of Aeschylus' Suppliants: the parodos

\author{
Marcus Mota \\ Universidade de Brasilia, Brasil \\ marcusmotaunb@gmail.com \\ (iD http://orcid.org/0000-0003-4745-8927
}

\section{Resumo:}

Neste artigo, apresento a escansão e uma análise preliminar da entrada do coro em cena na peça As Suplicantes de Ésquilo. O objetivo é providenciar material para estudos que levem em conta a relação entre texto, métrica e performance na interpretação de obras da dramaturgia ateniense antiga.

PalaVras-chaVe: Ésquilo, Dramaturgia, Métrica, Coro.

\section{Abstract:}

In this paper, I present the scansion and a preliminary analysis of the entrance of the choir in the scene in Aeschylus' The Supplices. The aim is to provide material for studies that take into account the relationship between text, metrics, and performance in the interpretation of works of ancient Athenian dramaturgy.

KEYWORDS: Aeschylus, Dramaturgy, Metrical Analysis, Choir.

\section{Passos iniciais}

A entrada ${ }^{1}$ do coro em As Suplicantes (párodo) segue a prática realizada em Sete contra Tebas: temos um módulo anapéstico que depois se desdobra em um conjunto estrófico. ${ }^{2} \mathrm{Ou}$ seja, temos em sucessão dois padrões de organização dos versos - um em homegeneidade métrica o outro heterométrico. Mas o que isso quer significar em termos de uma compreensão mais ampla do texto que chegou até nós?

A homogeneidade métrica aqui é percebida na reiterada utilizada de sistemas anapésticos. Os sistemas anapésticos, no contexto de entradas corais, indicam deslocamentos das entradas/ saídas laterais do teatro (eísodoi) para o espaço de cena. ${ }^{3}$ Por mais que possamos estabelecer algumas expectativas quanto à atualidade da atividade do coro a partir do metros, precisamos contar com outros indicativos, como o conteúdo das falas e a organização das estrofes. Em todo caso, a reconstituição da performance original é inacessível e nem deve guiar o intérprete.

O que sabemos é o seguinte: a regularidade dos anapestos em sua articulação binária aproxima-se de passos e movimentos simétricos e de grupo (Barris, 2011, p. 107). Essa regularidade é inserida um modo vocal que fica entre o canto e a fala, mais próximo de uma recitação, de uma fala entoada (West, 1982). Isso significa que não é performada como as partes faladas (trímetros iâmbicos), tendo uma melodia reduzida, a qual interage com a presença de acompanhamento de instrumento musical para orientar o ritmo e a frequência/ altura da notas.

Temos, pois, um grupo de agentes que sob um horizonte de um recorrente padrão rítmico adentra em sua fala-canto o campo de visão e audição do público acompanhados de instrumentos musicais.

A regularidade dos sistemas anapésticos é duplicada nas dipodias ou agrupamentos métricos. Como se pode ver na escansão abaixo, cada verso se divide em duas subfrases métricas, as quais portam o mesmo padrão métrico. Por isso, cada verso vem marcado como " 2 an". Ora se a unidade mínima do anapesto é uma base longa-breve-breve (-uu) e cada parte ou subfrase possui duas bases ou duas dipodias, temos assim que o 
dímetro anaspéstico explora essa tensão entre a regularidade do metro e suas possíveis variações, que no seguir da performance, são micro e significativas variações.

Essas variações são as seguintes:

\begin{tabular}{|l|l|l|l|}
\hline IDENTIFICAÇÃO & TEXTO & SINAIS & RITMO \\
\hline Anapesto & breve-breve-longa & uu- & S. J \\
\hline Dátilo & longa-breve-breve & -uu & JS $\delta$ \\
\hline Espondeu & longa-longa & -- & $\mathrm{J}$ \\
\hline
\end{tabular}

Ou seja, entre os versos 1 e 39 temos estratégias de ratificação de base anapéstica, que é invertida ou desacelerada a partir de suas isoformas (dátilo e espondeu). O que está em jogo é a produção de uma continuidade rítmica por meio de sutis variações do padrão. Para o público, ele ouve e vê corpos percussivos tomando conta da orquestra alinhando-se na recorrência de uma temporalidade que os define. ${ }^{4}$

Essas questões muitas vezes não são muito discutidas pois nas edições de textos as seções anapésticas recitadas ou não líricas (partes cantadas) não são escandidas. Como, de fato, as seções anapésticas podem ser traduzidas metricamente mais facilmente que as seções heterométricas das partes corais (estásimos), parece tácito que não há muito que discutir e analisar. Contudo, a prática de escansão aliada ao exame do texto demonstra que há estratégias bem específicas no uso de metros mesmo tão regulares como os anapestos.

Como se pode observar, o recurso a padrões facilmente perceptíveis e recorrentes não exclui variações e, disto, referências mais sutis. Além das isoformas do dátilo, temos outra estratégia que redefine a regularidade dos anapestos: temos a inserção de paremíacos, ou formas cataléticas do dímetro anapestos, como fechamento ou cláusula de subseções no párodo. Ou seja, temos dímetros anapestos interrompidos, para os quais falta alguma sílaba ou posição métrica que atualizaria o padrão em sua completude. Na escansão que se segue abaixo podemos ver este efeito que rompe com a expectativa de preenchimento de todas as posições métricas nos versos $4,7,10,13,18,22,25,32,36,39$.

Assim, a cada grupo de 2 e 3 versos em regularidade temos um contramovimento rítmico, um verso que não segue a recorrência completa dos dímetros anapésticos. Assim, o ritmo geral desse módulo anapéstico de entrada do coro é o um movimento regular que é reforçado algumas vezes e que depois se choca a contramovimento que o reorienta.

Este ritmo geral, em suas ondas de reiteração e interrupção, ultrapassa a fronteira linguística e textual do verso pode melhor ser apreendido quando aplicamos a ele algumas consequências do conceito de epiploké ou continuidade rítmica. $5 \mathrm{O}$ conceito é uma resposta a um problema comum em estudos rítmicos na versificação grega antiga: quando há ambivalência na interpretação dos registros métricos, temos muitas vezes posições ou metra em comum, as quais demonstram a prevalência do fluxo ou sucessão de unidades/ posições métricas. Assim, pela sequências trocaicas (base $-\mathrm{u}$ ) muitas vezes se confundem com sequências iâmbicas (base $u-)$, ou, como com em nosso caso, anapestos (uu-) com dátilos(-uu). Com isso, modulações rítmicas por padrões métricos assemelhados se constituem em uma chave para compreensão de modulações rítmicas em contextos heterométricos.

$\mathrm{O}$ que está em jogo na continuidade rítmica é a continuidade da performance. $\mathrm{O}$ ato de escansão é atomizante, trabalha com unidades isoladas, abstraídas de seu contexto coreográfico. Aplicando as consequências de se pensar esquemas rítmicos a partir das operações da epiploké, observa-se que, no contexto do módulo anapéstico de entrada do coro em As Suplicantes, a alternância entre movimento e contramovimento rítmico organiza a performance dos agentes em cena, inscrevendo-os no espaço vísivel e audível da orquestra. Assim, a escansão vista agora como um diagrama de forças e movimentos da atividade 
coral nos faculta o acesso às frases coreográficas e aos seus pontos redirecionamento das tensões que constroem o ritmo geral da cena.

Tal aplicação alteraria nosso modo de ler o texto: no lugar de pausas no final de cada verso, teríamos um jogo de continuidade ou sucessões dentro de outras continuidades:

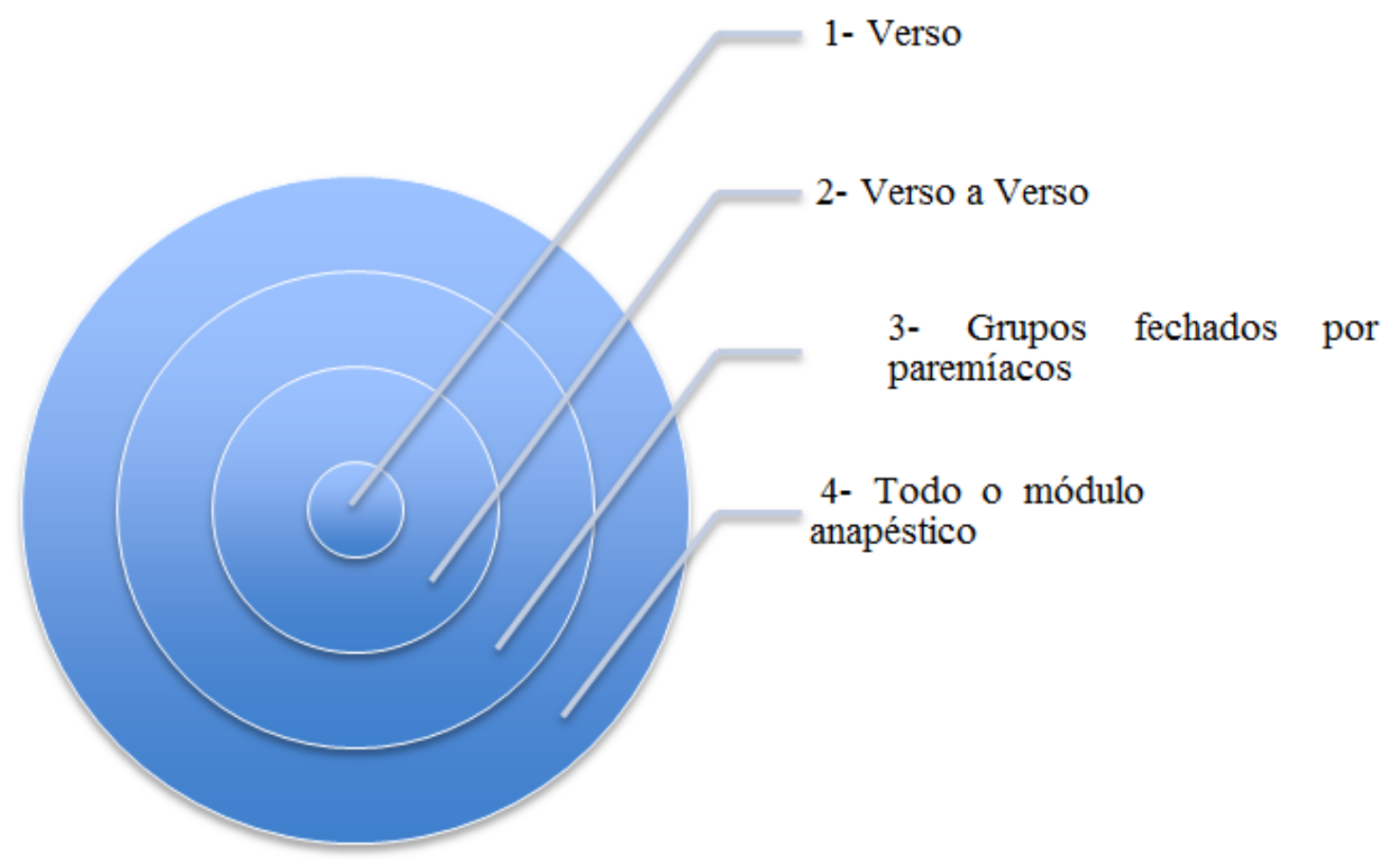

A imagem acima procura traduzir a correlação simultânea de diversas lógicas rítmicas que rumam em direção oposta à da desintegração analítica da escansão. No lugar de buscar referências cada vez menores, temos, pela correlação entre métrica e performance, a opção por soluções mais integrativas, que inserem as formas encontradas em níveis superiores de organização.

O resultado final é que vemos os versos se interligarem, formando uma linha sinuosa, um rastro de passos e sons, que é registrado no texto, mas que irrompe em sua totalidade na cena.

\section{A DANÇA DO CORO}

Após o módulo anapéstico, temos uma longa seção coral organizada em estrofes responsivas (vv. 40-155). É um outro processo de escrita e registro da atividade dos agentes corais. Temos 9 pares de sequências métrico-coreográficas, cada par ratificando uma mesma distribuição de ritmos. Assim, para o leitor audivosualmente orientado, temos que a cada par, o coro dança e canta duas vezes o mesmo arranjo métrico-coreográfico. Como a atividade coral é realizada à luz do dia, em situação frontal à platéia e a platéia se ergue em volta da área de atuação, é plausível concluir a partir dados que o coro primeiro apresenta sua coreografia voltando-se para um lado da audiência e depois para o outro.

A sucessão dos pares estróficos é a sucessão ou evolução da coreografia, como se vê por exemplo, nos desfiles do carnaval brasileiro (Mota, 2011a). ${ }^{6}$ Os pares se sucedem em arranjos métricos de diversos perfis, quanto ao número de versos e perfis métricos. Podemos ter combinações mais relacionadas a um perfil comum de metros, como os dátilos/ coriambos da primeira parte da atividade estrófica (vv. 40-111) e uma predominância mais iâmbica na segunda parte (vv. 112-175). Sob este amplo arco rítmico-coreográfico se 
sustentam as variações dentro dos versos e dos pares estróficos. $\mathrm{O}$ arco rítmico coreográfico, então, justapõe dois perfis métricos, relacionados movimentos e expectativas que são dançadas e cantadas em cena.

Desse modo, podemos observar que o desenho da atividade coral é uma exploração dramático-musical das transformações do páthos de um coro de mulheres que se coloca como frágeis, vítimas de perseguição, estrangeiras, mas também irrompem como um bando, um agrupamento militar. Elas são capazes de infligir ou receber violências. ${ }^{7}$ E é justamente na seção estrófica do párodo que esta ambivalência é exposta, intepretada coreograficamente pelo arco rítmico que divide a atividade do coro em duas sucessões com predominâncias rítmicas diversas: da invocação e memória épica do passado aos sofrimentos de agora temos a materialidade rítmica tradução a efetividade variacional dos afetos. Contudo este arco maior se sustenta nas réplicas ou apropriações que se dão dentro das estrofes. Segundo James Rasch (1981, p. 28), os três primeiros pares estróficos assim se articulam quanto aos seus referentes verbais:

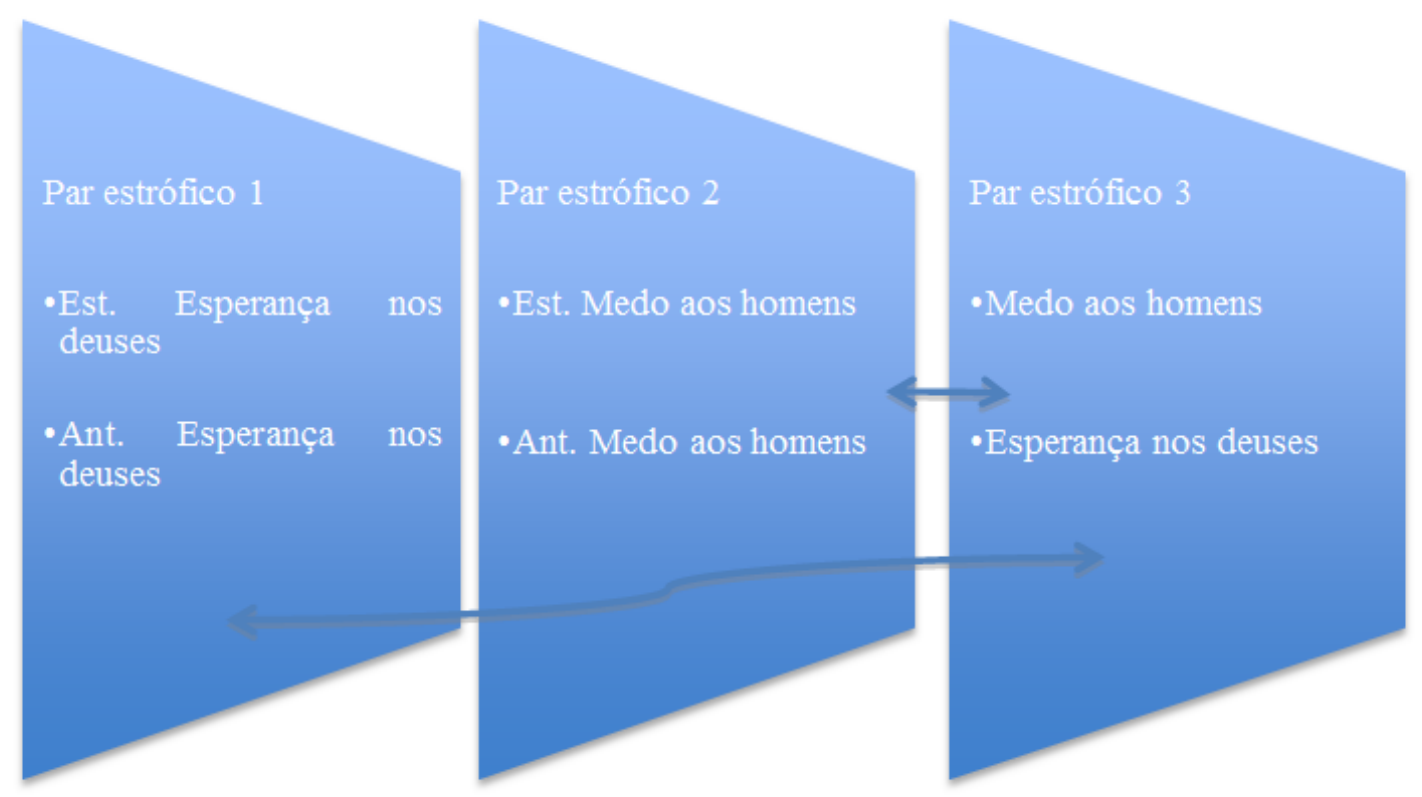

Sob um arco rítmico, temos as variações locais. A expectativa desse arco rítmico é construída a partir da tensão entre respostas emocionais contraditórias. E essas emoções contraditórias opõem não apenas pares estróficos entre si: como se vê no par estrófico 3, a estrofe alinha-se ao par estrófico 1, e a antiestrofe ao par estrófico 2.

Este feito dramatico-musical nos coloca diante de inúmeras possibilidades de compreender a correção entre texto, movimento e metro. Primeiro, há um desligamento entre o ritmo e conteúdo verbal, o que emancipa o estudo e compreensão da atividade performativa, vista agora não mais como uma ilustração audiovisual de palavras. A ilusão referencial do conteúdo verbal como foco da dramaturgia ateniense de fato é resultado de séculos de foco na textualidade em si, descontextualizada de suas condições de performance, como se a linguagem ela mesma gerasse seu próprio sentido. $\mathrm{O}$ que vemos aqui é um hábil dramaturgo que, experimentado no trabalho cênico, projeta para a audiência complexos meios de orientação da atividade do coro. $^{8}$

Assim, ouvimos uma dominância rítmica contra qual se contrapõe tensões afetivas em diversas distribuições (pares estróficos vs. pares estróficos, estrofe vs. antístrofe). 


\section{Projeções}

Há todo um trabalho a se fazer para tornar a atividade do coro algo mais compreensível, superando a imagem tradicional de um grupo estático de pessoas fazendo as mesmas coisas ao mesmo tempo, atuando como um jogral. Dos metros mais simples às organizações mais sofisticadas, observamos que o que de fato importa é a construção, a composição de uma performance.

O exemplo do párodo de As Suplicantes assinala que o arranjo dos valores rítmicos aproxima o texto de uma escrita coreográfica. Para a extração dessas informações, é preciso ter em mente o contexo performativo para orientar a intepretação dos dados métricos. O próximo passo é dançar, é propor projetos multidisciplinares, experimentos que testem convergências e divergências entre os dados filológicos e os feitos coreográficos. Assim, que sabe, consigamos daquilo que tem sido chamado de "terra incognita" do teatro (Mégevand, 2003, p. 105).

\section{Párodo de As Suplicantes, de Ésquilo9}

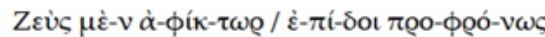

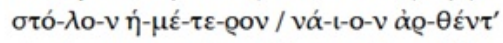

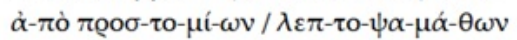

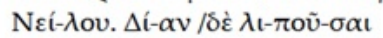

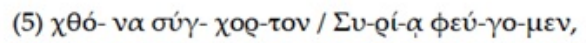

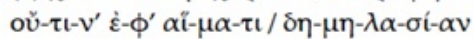

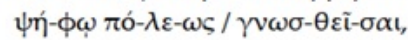

$\dot{\alpha} \lambda-\lambda^{\prime} \alpha \dot{v}-\tau 0-\gamma \varepsilon-v \varepsilon \tilde{I} / \phi v-\xi \alpha-v o-\rho i \alpha$, $\gamma \dot{\alpha}-\mu o-v$ Ai- $\gamma v \dot{u} \pi-\tau o v / \pi \alpha \hat{i}-\delta \omega-v \dot{\alpha}-\sigma \varepsilon-\beta \tilde{\eta} \tau^{\prime}$ (10) ò-vo- $\tau \alpha-\zeta o ́-\mu \varepsilon-v \alpha \mathrm{t} /<\delta \mathrm{t}-\alpha \dot{\alpha}-v o \mathrm{t}-\alpha v>$.

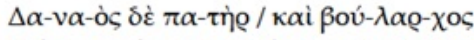

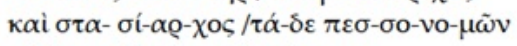
$\kappa \hat{v}-\delta \mathrm{l}-\sigma \tau^{\prime} \dot{\alpha}-\chi \dot{\varepsilon}-\omega v / \dot{\varepsilon}-\pi \dot{\varepsilon}-\kappa \varrho l-v \varepsilon v$

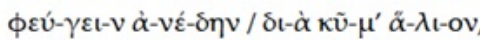
(15) $\kappa \varepsilon \dot{\lambda}-\sigma \alpha \mathrm{t}-\delta^{\prime}$ AQ- $\gamma o u \varsigma / \gamma \alpha \tilde{\mathrm{i}}-\alpha-v, \mathrm{o}-\theta \varepsilon v-\delta \dot{\eta}$

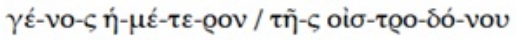

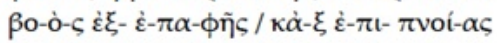
$\Delta \mathrm{t}-\mathrm{o}-\varsigma \varepsilon \dot{v}-\chi \hat{o}-\mu \varepsilon-v o v / \tau \varepsilon-\tau \dot{\varepsilon}-\lambda \varepsilon \sigma-\tau \alpha \mathrm{t}$.

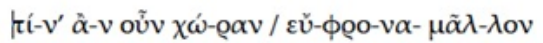
(20) $\tau \tilde{\eta} \sigma-\delta^{\prime} \dot{\alpha}-\phi \mathrm{t}-\kappa o i-\mu \varepsilon-\theta \alpha$

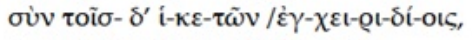

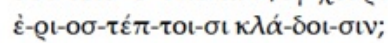

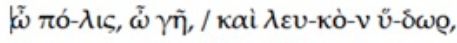

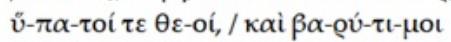
(25) $\chi \theta \dot{\theta}-v \mathrm{t}-\mathrm{ol} \theta \dot{\eta}-\kappa \alpha \varsigma / \kappa \alpha-\tau \varepsilon \dot{\varepsilon}-\chi \mathrm{ov}-\tau \varepsilon \varsigma$,
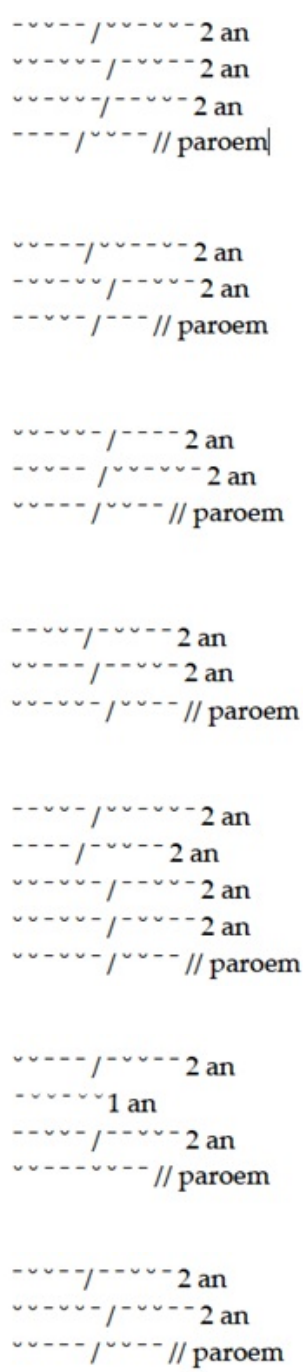


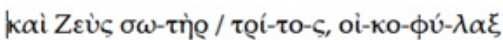

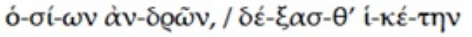

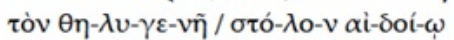
$\pi v \varepsilon v \dot{v}-\mu \alpha-\tau \iota \chi \hat{\omega}-\mathrm{Q} \alpha \varsigma \cdot / \alpha \grave{\alpha}-\sigma \varepsilon-v o-\pi \lambda \eta-\theta \tilde{\eta} \delta^{\prime}$

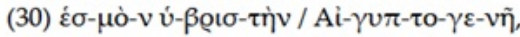

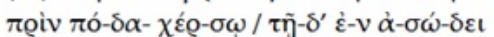

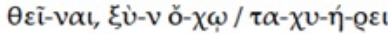

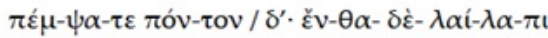

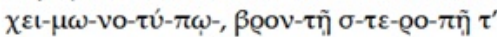

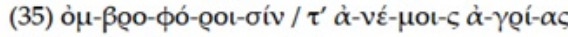

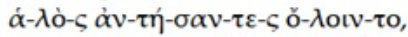

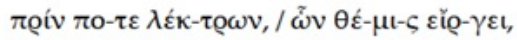
$\sigma \phi \varepsilon-\tau \varepsilon-\rho \mathrm{o}-\xi \dot{\alpha}-\mu \varepsilon-v o t / \pi \alpha-\tau \rho \alpha-\delta \dot{\varepsilon} \lambda-\phi \varepsilon \mathrm{t}-\alpha v$ $\tau \eta \dot{v} \delta^{\prime} \dot{\alpha}-\kappa o ́ v-\tau \omega v / \dot{\varepsilon}-\pi \mathrm{t}-\beta \tilde{\eta}-v \alpha \mathrm{t}$.

Est. 1 (40) vĩv $\delta^{\prime} \dot{\varepsilon}-\pi t-\kappa \varepsilon-\kappa \lambda o-\mu \varepsilon^{\prime}-v \alpha$ $\Delta \tilde{\mathrm{i}}-\mathrm{ov}-\pi \mathrm{o} \mathrm{Q}-\tau \mathrm{l}-\mathrm{v} \dot{\mathrm{v}}-\pi \varepsilon \mathrm{Q}-$

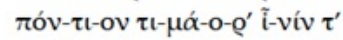

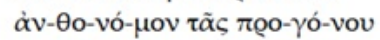

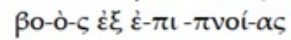

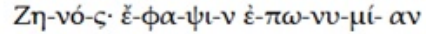

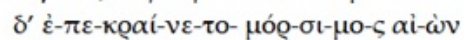
$\varepsilon \dot{v}-\lambda o ́-\gamma \omega-\varsigma,{ }^{\prime} \mathrm{E}-\pi \alpha-\phi o ́ v-\tau^{\prime} \dot{\varepsilon}-\gamma \varepsilon \dot{v} v-v \alpha-\sigma \varepsilon v$.

Ant. 1 ôv- $\tau^{\prime} \dot{\varepsilon}-\pi \mathrm{t}-\lambda \varepsilon-\xi \alpha-\mu \dot{\varepsilon}-v \alpha$,

(50) vṽ-v $\dot{\varepsilon} v-\pi 0 \mathrm{o}-0-v o ́-\mu o t \varsigma$ $\mu \alpha$ $\pi \varrho o ́ \sigma-\theta \varepsilon \pi \hat{c}-v \omega \nu \mu v \alpha-\sigma \alpha-\mu \dot{\varepsilon}-v \alpha$ $\tau \dot{\alpha} \tau \varepsilon v \tilde{v}-v \dot{\varepsilon}-\pi \mathrm{t}-\delta \varepsilon \dot{\varepsilon}-\xi \omega$

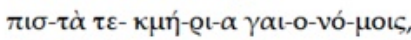

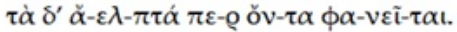

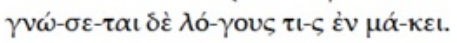

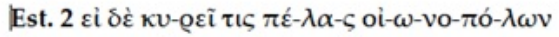

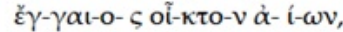

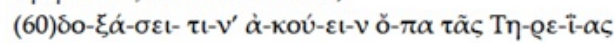

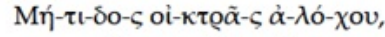

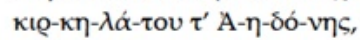

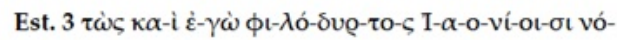

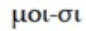

(70) $\delta \dot{\alpha} \pi-\tau \omega \tau \dot{\alpha}-v \dot{\alpha}-\pi \alpha-\lambda \dot{\alpha} v$

$\varepsilon \hat{i}-\lambda \mathrm{o}-\theta \varepsilon-\rho \tilde{\eta} \pi \alpha-\rho \varepsilon t-\dot{\alpha} v$

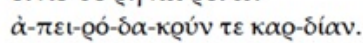

$\gamma \mathrm{o}-\varepsilon \delta-v \dot{\alpha} \delta^{\prime} \dot{\alpha} v-\theta \varepsilon-\mu i-\zeta \mathrm{c}_{0}-\mu \alpha \mathrm{t}$

$\delta \varepsilon t-\mu \alpha i-v o v-\sigma \alpha \phi i ́-\lambda o u \varsigma, \tau \tilde{\alpha} \sigma-\delta \varepsilon \phi v-\gamma \tilde{\alpha} \varsigma$

A- $\varepsilon-\rho i-\alpha-\varsigma \hat{\alpha}-\pi \dot{o} \gamma \tilde{\alpha} \varsigma$

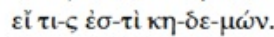

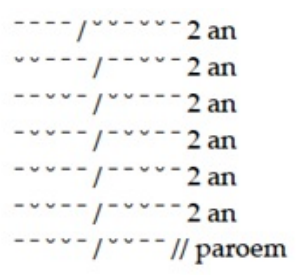

$\cdots$
$\cdots$
$\cdots$
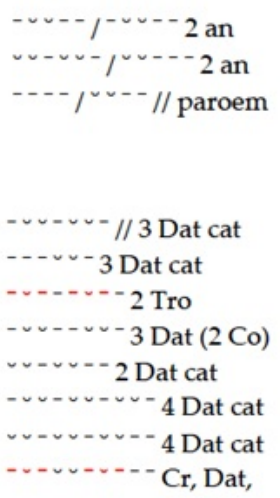

$-\cdots-\cdots$

- - -4

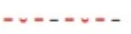

-ソง -

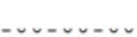

un-unu-

- - - von-

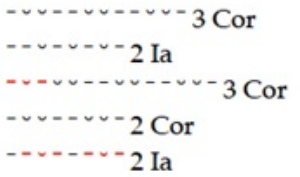

$-\cdots-\cdots-2$ Ia

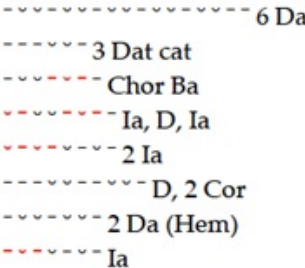




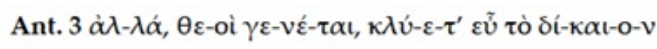

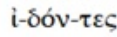

$\eta ๊-\beta \alpha \mu \eta \dot{~} \tau \dot{\varepsilon}-\lambda \varepsilon$-ov

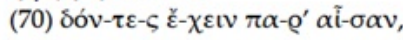

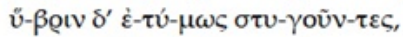

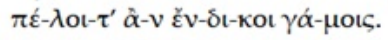

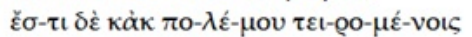

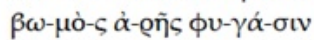

(85) 仓̊vं- $\mu \alpha, \delta \alpha t-\mu o ́-v \omega v \sigma \varepsilon \dot{\varepsilon}-\beta \alpha \varsigma$.

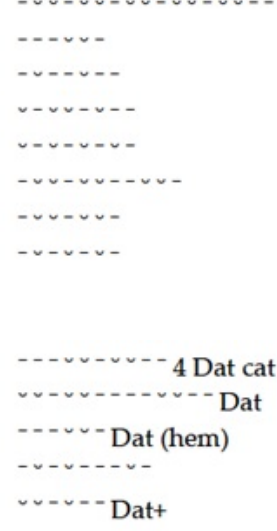

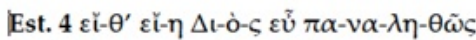
$\Delta \mathrm{t}-\mathrm{o}-\varsigma \mathrm{i}-\mu \varepsilon-\mathrm{eo}-\varsigma \cdot$ où $\varepsilon \dot{v}-\theta \dot{\eta}-\mathrm{e} \alpha-\tau \mathrm{o}-\varsigma \mathrm{\varepsilon}-\tau \dot{\chi} \chi-\theta \eta$. $\pi \alpha v-\tau \tilde{\alpha} \tau$

$\kappa \dot{v} v \sigma \kappa o ́-\tau \omega \mu \varepsilon-\lambda \alpha \dot{i}-v \alpha \underline{\alpha} \xi \dot{v} v \tau \tau \dot{v}-\chi \alpha$

$\mu \varepsilon$-อó- $\pi \varepsilon$ - $\sigma \sigma \iota \mathrm{l} \lambda \alpha$-oĩs.

Dat+

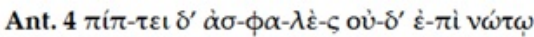

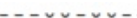

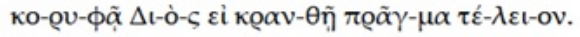

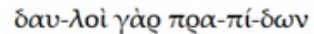

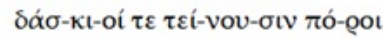

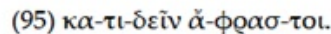

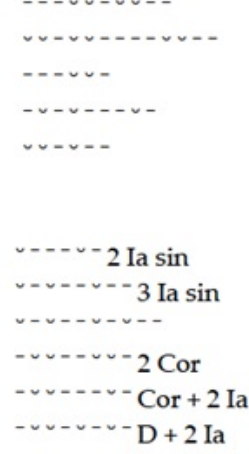

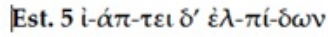

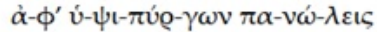

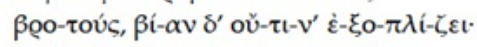

(100) $\pi \tilde{\alpha}-v \check{\alpha}-\pi 0-v o v \delta \alpha t-\mu o-v i-\omega v$.

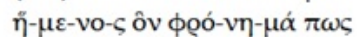

$\alpha \dot{v}-\tau \dot{0}-\theta \varepsilon-v \dot{\varepsilon}-\xi \dot{\varepsilon}-\pi \varrho \alpha-\xi \varepsilon-v \varepsilon \check{\mu} \mu$

$\pi \alpha-\varsigma \dot{\varepsilon}-\delta \varrho \dot{\alpha}-v \omega-v \dot{\alpha}-\phi^{\prime} \dot{\alpha} \gamma-v \tilde{\omega} v$.

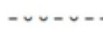

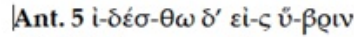

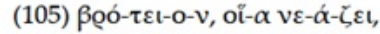

non

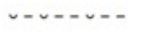

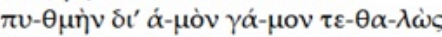

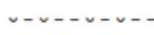

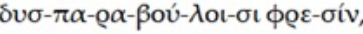

-

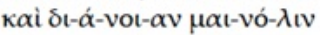

- non-

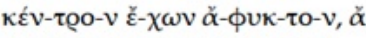

$\tau \alpha \delta^{\prime} \dot{\alpha}-\pi \dot{\alpha}-\tau \alpha \nu \mu \varepsilon-\tau \alpha \gamma$-voúc.

(n)

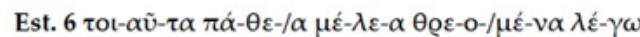

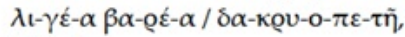

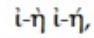

$i-\eta-\lambda \dot{\varepsilon}-\mu \mathrm{ol}-\sigma \mathrm{t}-\nu \dot{\varepsilon} \mu-\pi \varrho \varepsilon-\pi \tilde{\eta} \cdot$

(115) $\zeta \tilde{\omega}-\sigma \alpha \gamma$ ó-oเৎ $\mu \varepsilon \tau \mathrm{\tau}-\mu \tilde{\omega}$.

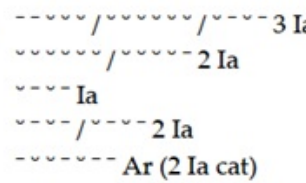

$i-\lambda \varepsilon-\tilde{\omega}-\mu \alpha t \mu \dot{\varepsilon}-v$ A- $\pi \hat{i}-\alpha v \beta o \tilde{v}-v t v$

$\kappa \alpha \varrho-\beta \tilde{\alpha}-v \alpha \delta^{\prime} \alpha \dot{v}-\delta \dot{\alpha}-v \varepsilon \tilde{v}, \gamma \tilde{\alpha}, \kappa o v-v \varepsilon i \bar{l}$.

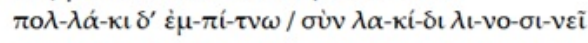

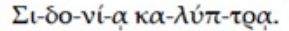

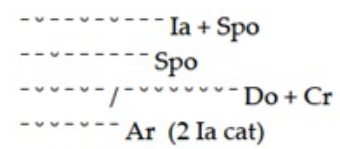

Ant. $6 \theta \varepsilon$-oī $\delta^{\prime} \dot{\varepsilon}-v \alpha-\gamma \varepsilon-\alpha \tau \dot{\varepsilon}-\lambda \varepsilon-\alpha \pi \varepsilon-\lambda o-\mu \varepsilon \dot{\varepsilon}-v \omega v \kappa \alpha-$ $\lambda \tilde{\omega} s$

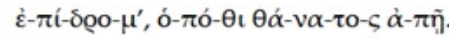

i- $\dot{\omega} \mathbf{i}-\dot{\omega}$,

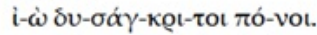

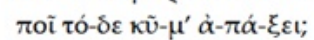

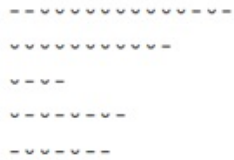


$\mid i-\lambda \varepsilon-\tilde{\omega}-\mu \alpha t \mu \dot{\varepsilon}-v$ A- $\pi i-\alpha v \beta$ oṽ-viv $\kappa \alpha \varrho-\beta \tilde{\alpha}-v \alpha \delta^{\prime} \alpha \dot{v}-\delta \dot{\alpha}-v \varepsilon \tilde{v}, \gamma \tilde{\alpha}, \kappa o v-v \varepsilon \tilde{c}$.

Ia + Spo

$\pi \mathrm{o} \lambda-\lambda \dot{\alpha}-\kappa \mathrm{t} \delta^{\prime} \dot{\varepsilon} \mu-\pi \hat{i}-\tau v \omega / \sigma \dot{v} v \lambda \alpha-\kappa \hat{i}-\delta \mathrm{t} \lambda \mathrm{t}-v \mathrm{o}-\sigma \mathrm{t}-v \varepsilon \tilde{\imath}$

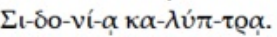

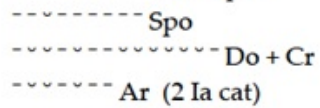

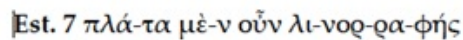

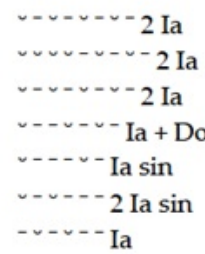

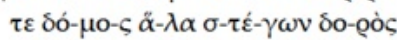

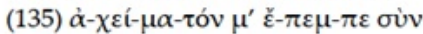

$\pi v o-\alpha i \tilde{c} \cdot o \dot{ } \cdot \delta \dot{\varepsilon} \mu \varepsilon \dot{\varepsilon} \mu-\phi o-\mu \alpha \mathrm{l}$.

$\tau \varepsilon-\lambda \varepsilon v-\tau \dot{\alpha} \varsigma \delta^{\prime} \dot{\varepsilon} \nu-\chi \varrho o ́-\nu \omega$

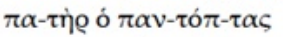

$\pi \varrho \varepsilon v-\mu \varepsilon-v \varepsilon \tilde{i} \varsigma$ $\kappa \tau i ́-\sigma \varepsilon-1 \varepsilon v$.

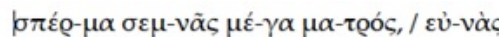

(140) $\alpha \hat{\alpha} v-\delta \varrho \tilde{\omega} v, \hat{\varepsilon} \varepsilon$,

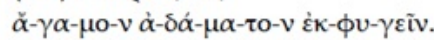

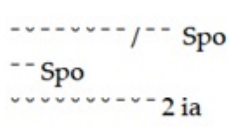

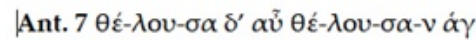

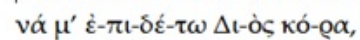

(145) $\check{\varepsilon}-\chi o v-\sigma \alpha \sigma \varepsilon \dot{\mu} \mu-v^{\prime} \dot{\varepsilon}-v \dot{\omega}-\pi \mathrm{I}^{\prime} \dot{\alpha}-$

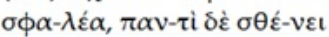

$\delta \mathrm{t}-\omega \gamma-\mu \mathrm{oī \varsigma} \delta^{\prime} \dot{\alpha} \sigma-\phi \alpha-\lambda \varepsilon \dot{\varepsilon} \alpha$

$\dot{\alpha} \delta-\mu \tilde{\eta}-\tau 0-\varsigma \dot{\alpha} \delta-\mu \eta \dot{\eta}-\tau \alpha$

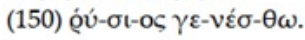

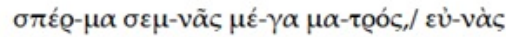
$\grave{\alpha} v-\delta \varrho \omega \tilde{\omega} v, \hat{\varepsilon} \tilde{\varepsilon}$,

$\check{\alpha}-\gamma \alpha-\mu \mathrm{o}-v \dot{\alpha}-\delta \dot{\alpha}-\mu \alpha-\tau 0-v \dot{\varepsilon} \kappa-\phi v-\gamma \varepsilon \tilde{\varepsilon} v$.

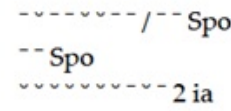

Est. $8 \varepsilon \dot{\varepsilon} \delta \dot{\varepsilon} \mu \eta \dot{,}, \mu \varepsilon-\lambda \alpha v-\theta \dot{\varepsilon} \varsigma$

(155) $\dot{\eta}-\lambda t-o ́ \kappa-\tau v-\pi o v \gamma \hat{\varepsilon}-$-vo

tòv $\gamma \alpha \dot{\alpha}-\mathrm{t}-\mathrm{ov}$

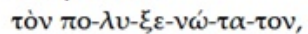

$Z \tilde{\eta}-v \alpha \tau \tilde{\omega} \nu \kappa \varepsilon-\kappa \mu \eta-\kappa o ́-\tau \omega \nu$

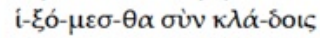

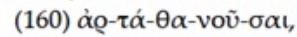

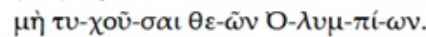

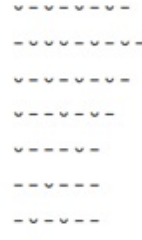

$\tilde{\alpha} Z$ Zńv, I-oũ $\zeta \cdot i-\dot{\omega} \mu \tilde{\eta}-v ı \varsigma$ $\mu \alpha \dot{\sigma} \sigma-\tau \varepsilon \varrho^{\prime} \dot{\varepsilon} \kappa \theta \varepsilon-\tilde{\omega} v$.

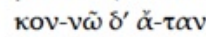

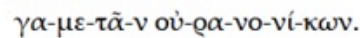

(165) $\chi \alpha-\lambda \varepsilon-\pi \circ \tilde{0} \gamma \dot{\alpha} \varrho$ हों

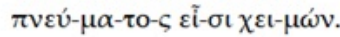

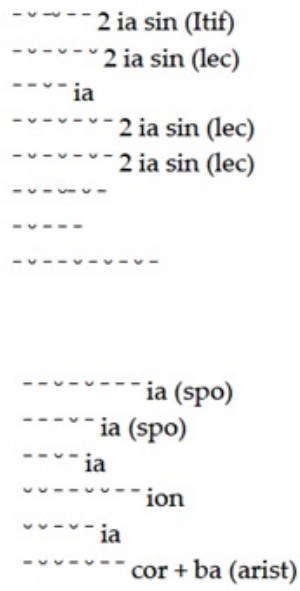

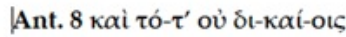

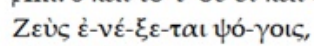

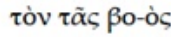

(170) $\pi \alpha \tilde{\imath}-\delta^{\prime} \dot{\alpha}-\tau \mathrm{l}-\mu \alpha^{\prime}-\sigma \alpha \varsigma, \tau \dot{0}-v \alpha \dot{v}-$

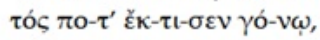

vข̃v

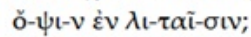

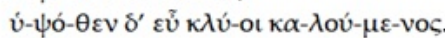

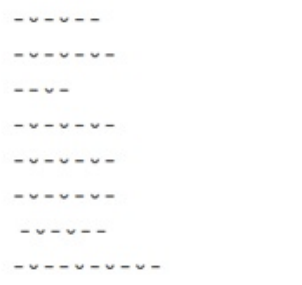




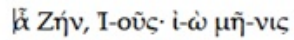

$$
\begin{aligned}
& \mu \alpha \dot{\sigma} \sigma-\tau \varepsilon \varrho^{\prime} \dot{\kappa} \kappa \theta \varepsilon-\tilde{\omega} v \text {. }
\end{aligned}
$$

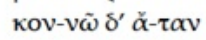

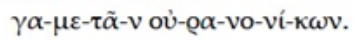

$$
\begin{aligned}
& \chi \alpha-\lambda \varepsilon-\pi \circ \tilde{v} \gamma \dot{\alpha} \varrho \dot{\varepsilon} \kappa
\end{aligned}
$$

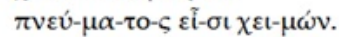

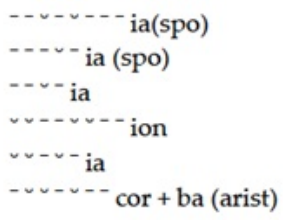

\section{REFERÊNCIAS}

Cole, T. (1988). Epiploke: Rhythmical Continuity and Poetic Structure in Greek Lyric. Cambridge: Harvard University Press.

Dale, A. M. (1968). The Lyric Metres of Greek Drama. Cambridge: Cambridge University Press.

Dale, A. M. (1969). Collected Pappers. Cambridge: Cambridge University Press.

Fleming, T. (2007). The Colometry of Aeschylus. Amsterdã: Adolf M. Hakkert Editore.

Gentili, B. (1951). La metrica dei Greci. Messina/Fireze: Casa Editrice G. D’Anna.

Guerra, A. G. (1997). Manual de Métrica Grega. Madrid: Ediciones Clássicas.

Lourenço, R. (2011). Para uma terminologia portuguesa da métrica grega. Boletim de Estudos Clássicos 55, 17-55.

Maas, P. (1962). Greek Metre. Oxford: Oxford University Press.

Martinelli, M. C. (1997). Gli Strumenti del Poeta. Bologna: Cappelli Editore.

Masqueray, P. (1899). Traité de Métrique Grecque. Paris: Librarie C. Klincksieck.

Mégevand, M. (2003). L'éternel retour du chœur. Littérature 131, 105-122.

Mota, M. (2008). A dramaturgia musical de Ésquilo: Investigaçôes sobre composição, realização e recepção de fiç̧ão audiovisual. Brasilia: Editora Universidade de Brasilila.

Mota, M. (2011). Metro e representação: geração de arquivos sonoros e midi a partir de textos da tragédia grega. En: ANAIS 7SIMCAM, 254-266.

Mota, M. (2011a). From Text to Performance: Ancient Greek Drama and Brazilian Carnival. In: Languages and Cultures in Contact and Contrast: Historical and Contemporary Perspectives (pp. 239-244). Atenas: ATINER.

Mota, M. (2012). Ouvir e dançar ritmos: Experimentos com metros da tragédia grega. Clássica. Revista Brasileira de Estudos Clássicos v. 25, 133-148.

Mota, M. (2013). Nos passos de Homero. Ensaios sobre performance, filosofia, música e dança a partir da antiguidade. São Paulo: Annablume.

Mota, M. (2018). Performance e estudos clássicos: proposta de seminários interdisciplinares. Em L. Oliveira y A. Bacelar (Ed.), Cenas Poéticas, Filosóficas e Musicais (pp. 215-248). São Paulo: Annablume.

Mota, M. (2018a). Ésquilo: dramaturgia e repertório uma discussão preliminar. Revista Clássica 31 (2), 75-88. Mota,

M. (2018b). Vida de Ésquilo. Tradução. Rónai: Revista de Estudos Clássicos e Tradutórios 6, 52-62.

Mota, M. (2019). Ritmos/Pés Métricos em Performance: Notas sobre a Dramaturgia Grega Antiga a partir de exemplos em Ésquilo. Revista VIS. Revista do Programa de Pós-Graduação em Arte 18 (1), 21-49.

Rasch, J. N. (1981). Meter and Language in the Lyrics of the Suppliants of Aeschylus. Nova York: Arno Press.

Raven, D. S. (1962). Greek Metre. Londres: Faber and Faber.

Sandin, P. (2005). Aeschylus' Supplices. Introduction and Commentary on vv 1-523. Lund: Symmachus.

Schroeder, O. (1907). Aeschyli Cantica. Stutgart: Teubner.

Steinrck, M. (2007). À quoi sert la métrique? Grenoble: Jérme Millon.

West, M. L. (1982). Greek Metre. Oxford: Oxford University Press.

West, M. L. (1990). Aeschylus. Tragoediae cum incerti poetae Prometheo. Leipzig: Teubner. 


\section{Notas}

1 Os materiais de escansão foram elaborados para seminário multidisciplinar que, nas fronteiras entre Estudos Clássicos e Estudos Teatrais, objetivou o estudo e reperformance de seções de As Suplicantes de Ésquilo e de materiais do mito das Danaides (cf. Mota, 2018). Aqui apresento apenas parte dessa escansão.

2 Para o texto, valho da edição West (1990).

3 Para uma análise mais detida do párodo e de sua métrica, cf. Mota (2008). Para questões do espaço de representação do Teatro de Dioniso, Mota (2017). Para um exame dos metros em performance na dramaturgia ateniense, Mota (2019).

4 Discuto mais sobre isso em Mota (2012).

5 Sobre o tema, cf. Cole (1988).

6 Textos de minha autoria se encontram disponíveis em https://brasilia.academia.edu/MarcusMota. Acesso 20072019.

7 Para mais detalhes, cf. Mota (2008).

8 Sobre o trabalho do dramaturgo, cf. Mota (2018a y 2018b).

9 Para as abreviaturas, sigo nomenclatura apresentada em Lourenço (2011) e siglas em West (1990). Para a escansão, além da edição de WEST já citada, vali-me de Masqueray (1899), Schroeder (1907), Gentili (1951), Raven (1962), Maas (1962), Dale (1968), Dale (1969), Rasch (1981), Guerra (1997), Sandin (2005), Martinelli (1997), Fleming (2007). Em vermelho são marcadas posições métricas ambivalentes, discutíveis, sem consenso entre metricistas. 